\title{
Economic evaluation of cardiac magnetic resonance with fast-SENC in the diagnosis and management of early heart failure
}

\author{
John E. Schneider ${ }^{*}$ and Ivana Stojanovic
}

\begin{abstract}
Introduction: Heart failure (HF) is a major public health concern, prevalent in millions of people worldwide. The most widely-used HF diagnostic method, echocardiography, incurs a decreased diagnostic accuracy for heart failure disease progression when patients are asymptomatic compared to those who are symptomatic. The purpose of this study is to conduct a cost-effectiveness analysis of heart failure diagnosis comparing echocardiography to a novel myocardial strain assessment (Fast-SENC), which utilizes cardiac-tagged magnetic resonance imaging.

Methods: We develop two models, one from the perspective of payers and one from the perspective of purchasers (hospitals). The payer model is a cost-effectiveness model composed of a 1-year short-term model and a lifetime horizon model. The hospital/purchaser model is a cost impact model where expected costs are calculated by multiplying cost estimates of each subcomponent by the accompanying probability.

Results: The payer model shows lower healthcare costs for Fast-SENC in comparison to ECHO $(\$ 24,647$ vs. $\$ 39,097)$ and a lifetime savings of $37 \%$ when utilizing Fast-SENC. Similarly, the hospital model revealed that the total cost per HF patient visit is $\$ 184$ for ECHO and $\$ 209$ for Fast-SENC, which results in hospital contribution margins of $\$ 81$ and $\$ 115$, respectively.

Conclusions: Fast-SENC is associated with higher quality-adjusted life years and lower accumulated expected healthcare costs than echocardiogram patients. Fast-SENC also shows a significant short-term and lifetime costsavings difference and a higher hospital contribution margin when compared to echocardiography. These results suggest that early discovery of heart failure with methods like Fast-SENC can be cost-effective when followed by the appropriate treatment.
\end{abstract}

Keywords: Heart failure, Cardiac magnetic resonance imaging, Cost-effectiveness, Hospital value analysis, Markov model

\section{Introduction}

Heart failure (HF) is a major public health concern, with a prevalence of $1-2 \%$, or more than 5.8 million people in the U.S. and over 23 million worldwide $[1,2]$. Each year in the U.S., more than 550,000 individuals are diagnosed with HF for the first time [3]. The increasing prevalence is due in part to the aging of the population, increasing rates of obesity and diabetes, and more generally the prolongation of the lives of cardiac patients [4-7].

* Correspondence: John.Schneider@avalonecon.com

Avalon Health Economics, 26 Washington Street, 3rd Floor, Morristown, NJ 07960, USA

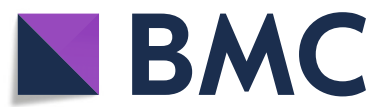

The lifetime risk of developing HF is about $20 \%$, and that risk is the same at age 40 and age 80 [8].

There are approximately one million annual hospitalizations in the U.S. due to HF [4]. Heart failure is the leading cause of all hospitalizations and readmissions in older people, and affects $6-10 \%$ of people over the age of 65 [9]. After hospitalization, HF prognosis generally worsens [10]. Although the outcomes for ambulatory HF patients with a reduced ejection fraction have improved with the innovation of drug and device therapies, hospitalized HF patients continue to experience high post-discharge mortality and readmission rates [4]. Heart failure is also associated with a disproportionately higher use of ambulatory

(C) The Author(s). 2019 Open Access This article is distributed under the terms of the Creative Commons Attribution 4.0 International License (http://creativecommons.org/licenses/by/4.0/), which permits unrestricted use, distribution, and reproduction in any medium, provided you give appropriate credit to the original author(s) and the source, provide a link to the Creative Commons license, and indicate if changes were made. 
services, including emergency department and clinic visits, especially towards the end of life $[8,11,12]$. Nonetheless, many of HF-related hospitalizations can be considered "avoidable", either due to poor disease management generally or lack of "systems approach" to early diagnosis and treatment (e.g., providing adequate outpatient treatment to avoid hospitalizations) [13-17]. As such, the economic burden of Stage B HF is high and will likely continue to grow over time.

Echocardiography, as the most widely-used diagnostic method, measures left ventricular (LV) ejection fraction (EF) as a global index of ventricular systolic function. However, LVEF is affected by the ventricular geometry and loading condition which may remain unchanged in affected patients until the underlying disease process is advanced [18]. Moreover, LVEF, by itself, has been shown to be a poor indicator of HF disease progression, with a positive predictive value of only $72 \%$ [19]. The key to diagnosis of Stage B HF is evidence of cardiac structural remodeling or functional abnormalities in the absence of any HF symptoms [20].

There is recent and developing literature describing the clinical utility of myocardial strain assessment using cardiac-tagged magnetic resonance imaging (CMR) in conjunction with algorithms and software to augment the clinical utility of CMR [21]. For example, strain-encoded CMR (Fast-SENC, known commercially as MyoStrain ${ }^{\mathrm{Tu}}$ software developed by Myocardial Solutions, Inc., Raleigh, $\mathrm{NC}$ ) is a heart assessment test that provides myocardial strain measurements within 10 min without using contrast or radiation, and quantifies myocardial strain across 37 regions of the heart that show heart dysfunction on both a global and regional level. By quantifying strain metrics and visualizing the affected regions of the heart, FastSENC can identify dysfunction resulting from a broad range of cardiac diseases, including stenosed blood vessels, ruptured atherosclerotic plaque, or microvascular diseases, allowing physicians to make more informed clinical decisions [22]. Fast-SENC also offers previously unavailable cardiac function data to determine the subclinical effects on the heart of $\mathrm{HF}$ and associated treatments, allowing physicians to make more timely and effective treatment decisions. In this study, we conduct a cost-effectiveness analysis of HF diagnosis using Fast-SENC, assessing the economic impact of the improved diagnostic capability of Fast-SENC, taking into account changes in the clinical pathway and resources associated with "Fast-SENC-guided" HF treatment compared to the standard of care (i.e., echocardiography).

\section{Methods}

We developed models from the perspectives of payers (i.e., insurance plans) and from the perspective of purchasers (i.e., hospitals and health systems). The payer perspective model is a cost-effectiveness analysis that follows methods recommended by the U.K. National Institute for Clinical Excellence (NICE) as well as guidelines developed by the International Society for Pharmacoeconomics and Outcomes Research (ISPOR).Given insurance switching by patients over time, payers covering initial costs may benefit at all any downstream cost offsets $[23,24]$. Consequently, we composed an insurance payer model of a 1-year short term model and a lifetime horizon long term model.

Patient population lifetime costs were modeled with Markov processes. The hypothetical patient cohort is on average 64 years and its characteristics are based on a clinical trial cohort with a pretest probability for early HF of $20 \%$, as determined by a Charlson Comorbidity Index score [25, 26]. The hospital model is a 1-year model and the hypothetical patient cohort matches that one of the payer model.

\section{Payer model}

We developed the HF model framework based on information obtained from a review of the published literature containing information on the management and clinical outcomes of HF patients. The model assumed patients will undergo the standard HF screening assessing patient history and physical examination. Scoring three or higher on the Charlson Comorbidity Index scale during the initial examination, would add a diagnostic assessment of the heart with either echocardiography (ECHO) or Fast-SENCT to assess the long-term effects of early HF diagnosis with imaging modalities, the model accounted for a lifetime of costs and health effects across a hypothetical cohort of patients using a Markov model of HF disease progression [27-33]. The model used transition probabilities (shown in Additional file 1)to allow patients to transition between disease states over time. Each disease state was assigned a time-dependent utility and cost. Disease state transition probabilities are based on literature and consultations with clinical experts. Based on discussions with clinical experts, we assumed that the initial diagnostic effect is associated with an initial treatment effect, and that the initial treatment effect impacts subsequent disease state transition probabilities (i.e., through better protection of heart muscle during the episode of care following diagnosis).

The long-term expected health effects and costs of imaging modality strategies were calculated by multiplying the total time spent in each disease state by the utilities and costs corresponding to these states, respectively. The model employed 1-year time cycles and the simulation stopped when all patients in the hypothetical cohort died. The simulation begins with a short-term model that divides HF patients based on the imaging modality. The group that got early HF diagnosis with Fast-SENC 
or went undiagnosed in the $\mathrm{ECHO}$ group. With a diagnostic accuracy of $100 \%$, Fast-SENC allowed for patients to be treated with medications and recommended lifestyle modifications, reducing the incident rate of HF hospitalizations [16]. ECHO is the imaging standard of care in assessing early deterioration of LV function, however it is not able to detect dysfunction within the heart wall. Thus, ECHO will fail to diagnose the early signs of HF.

The Markov decision analytic model was structured with five disease states, including the beginning state of HF (Stage B), Stage C, Stage C+, Stage D and the death state as described in the ACCF/AHA Guideline for the Management of Heart Failure (Fig. 1) [34]. In the model not all patients started in the early HF Stage B state, as some patients were assumed to have already had a HF hospitalization and thus started in the Stage C. Individuals without $\mathrm{HF}$ at the time of the screening stayed in the suspected HF stage, (i.e., Stage B) until they developed HF and progressed further into one of the more advanced Markov disease states. Patients could then be detected by the subsequent annual screening similar to the individuals with HF who were missed with the initial screening. Finally, all individuals could die from causes unrelated to HF, while HF patients could also die due to HF. In the model, health effects were determined by calculating expected life-years and quality adjusted life-years (QALY). Utilities for each of the five disease states were based on the HF patient cohort reported by Banz et al. [35].

\section{Data}

Fast-SENC is a relatively new technology, therefore clinical data required to populate the model was collected from the HF literature, including health statistics and expert opinions. Data inputs for the payer are shown on
Table 1, and data inputs for the hospital model are shown on Table 2. The expected treatment effect for patients who are diagnosed with early HF and treated with beta-blockers and ACE inhibitors was estimated from a clinical study reported by Pfeiffer et al. The risk of death from causes other than HF based was based on age- and gender- life tables for the U.S. individuals, whereas risk from death from HF was estimated based on the study of 3752 individuals in the Cardiovascular Health Study [25]. The costs taken into account in the model included HF hospitalizations, annual pharmacology costs, annual office visits, imaging, and procedural intervention costs, shown in Tables 1 and 2. Utility values were also based on the literature and assigned to each disease state by the New York Heart Association (NYHA) classification based on clinical guidelines [35, 36].

\section{Key assumptions}

The model assumed that patients entered into two hypothetical cohorts when they were assessed for HF with either Fast-SENC or ECHO. With the ability to detect changes and deterioration of the heart wall, Fast-SENC was assumed to be able to allow clinicians to start patients with medical therapy and lifestyle changes. Michalsen et al. concluded that $54.2 \%$ of $\mathrm{HF}$ hospitalization could be avoidable if patients received treatment sooner, adhered to prescribed medications and changed their lifestyles [16]. Thus, we assumed that using Fast-SENC a 27.1\% reduction in HF hospitalizations (i.e., assuming half of Michalsen et al.'s reported avoidable hospitalizations) could be achieved with early HF diagnosis.

The model assumed that disease-state costs do not vary across cohorts, and that at each state the following costs

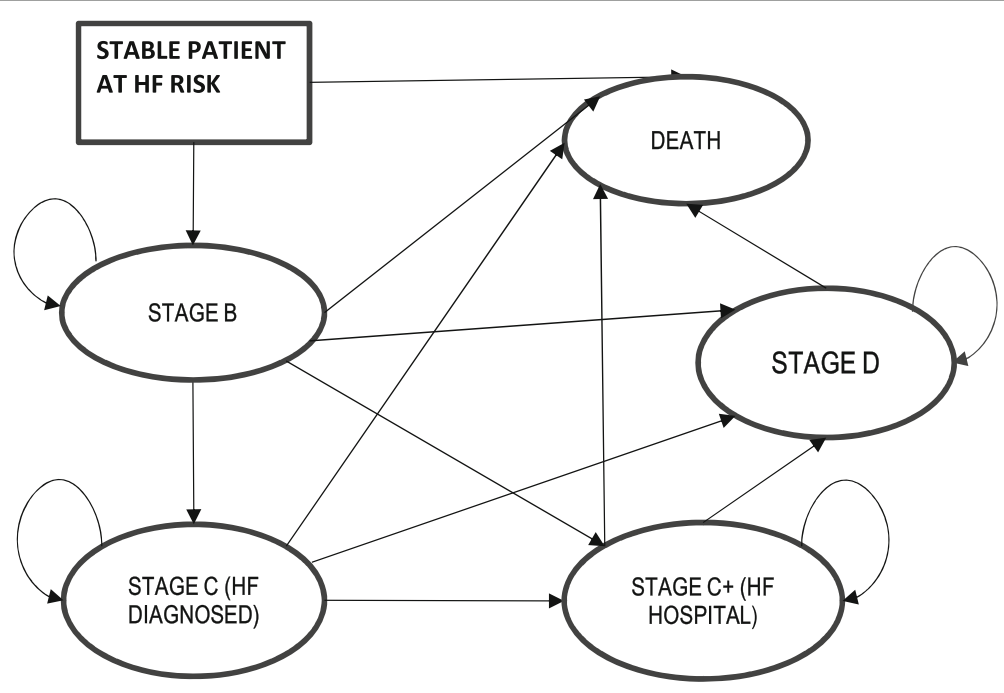

Fig. 1 Markov model representing HF disease progression 
Table 1 Clinical Input Data for Payer Model

\begin{tabular}{|c|c|c|}
\hline Variables & Base-case & Article References \\
\hline Average age of cohort & 64 & [25] \\
\hline $\begin{array}{l}\text { Prevalence of HF stage B+ after } \\
\text { Charlson screening }\end{array}$ & 0.2 & Pretest assumption \\
\hline Outcomes discount rate & $3 \%$ & {$[40]$} \\
\hline SENC Rest Diagnostic Accuracy & 1 & [18] \\
\hline $\begin{array}{l}\text { 4-year progression in prevalence } \\
\text { of stage B }\end{array}$ & 0.154 & [41] \\
\hline \multicolumn{3}{|l|}{$\mathrm{ECHO}$ at rest } \\
\hline Stage $C$ & $4.27 \%$ & {$[42]$} \\
\hline Stage $\mathrm{C}_{+}$ & $4.55 \%$ & {$[42]$} \\
\hline Stage D & $4.50 \%$ & {$[26]$} \\
\hline Mortality & $6.94 \%$ & {$[42]$} \\
\hline \multicolumn{3}{|l|}{ Fast-SENC } \\
\hline Stage C & $3.11 \%$ & {$[42]$} \\
\hline Stage $\mathrm{C}+$ & $3.32 \%$ & {$[42]$} \\
\hline Stage D & $3.28 \%$ & Assumption \\
\hline Mortality & $5.43 \%$ & {$[42]$} \\
\hline $\begin{array}{l}\text { Percent of Heart Failure Hospitalizations } \\
\text { that are Preventable }\end{array}$ & $54.20 \%$ & [16] \\
\hline $\begin{array}{l}50 \% \text { reduction in preventable } \\
\text { hospitalization for SENC }\end{array}$ & $27.10 \%$ & Assumption \\
\hline HF hospitalization (annualized rate) & $32.02 \%$ & [17] \\
\hline 30-day HF rehospitalization & $26.90 \%$ & [43] \\
\hline
\end{tabular}

were incurred: (1) Stage B costs included annual physician visits, medication and diagnostic imaging; (2) Stage C costs included probability of intervention procedural costs; (3) Stage C+ included costs of HF hospitalization and the probability of rehospitalization; and (4) Stage D costs

Table 2 Resource Use Input Data for Payer Model

\begin{tabular}{lll}
\hline Variables & Base-case & Article References \\
\hline Fast-SENC MRI rest & $\$ 324$ & Medicare Fee Schedule \\
ECHO rest & $\$ 231$ & Medicare Fee Schedule \\
Contrast medicine & $\$ 45$ & Assumption \\
Drug costs PMPM for HF patients & $\$ 367$ & {$[44]$} \\
$\begin{array}{l}\text { Drug costs for advanced HF } \\
\text { patients }\end{array}$ & $\$ 580$ & {$[44]$} \\
$\begin{array}{l}\text { Mean cost of HF hospitalization } \\
\text { as primary diagnosis }\end{array}$ & $\$ 17,654$ & {$[45]$} \\
$\begin{array}{l}\text { Mean cost of HF hospitalization } \\
\text { as secondary diagnosis }\end{array}$ & $\$ 25,325$ & {$[45]$} \\
$\begin{array}{l}\text { Mean cost of HF hospitalization } \\
\text { Cost of Ischemic heart disease }\end{array}$ & $\$ 23,077$ & {$[45]$} \\
hospitalization & $\$ 14,989$ & {$[45]$} \\
Outpatient/Office visit & $\$ 165$ & Medicare Data Milliman \\
Cost discount rate & & Report \\
\hline
\end{tabular}

included palliative care and the likelihood of mechanical assist device implantation. Total disease state costs were multiplied by the appropriate transition probabilities $(1-$ transition probability) for each corresponding state to calculate the expected cost across cohorts. Finally, we assumed that the quality of life values did not differ among the cohorts. Average utility for all patients in Stage B heart failure was 0.80 for patients with mild HF, 0.65 for advanced HF patient and 0.30 for all patients in the last stages of the HF disease [35]. Health utilities were multiplied by the percentage of patients alive in each cohort to derive the average quality-adjusted life years (QALYs) for the cohort in each year of the model.

\section{Hospital model}

The hospital model is a linear one-year accounting model estimating total cost of Fast-SENC and ECHO imaging ownership, imaging facility and technical costs, test costs, and medication costs. The hospital model aims to provide per patient hospital contribution margin for all $\mathrm{HF}$ patients diagnosed early with either ECHO or Fast-SENC. Fast-SENC costs were calculated using an initial purchasing price for the software, an ongoing maintenance cost, and a per-test fee. The hospital model calculated hospital profit contribution margins per HF diagnostic test, comparing Fast-SENC and ECHO. The model quantified lost annual marginal revenue for patients admitted for unplanned hospitalizations and also quantified the additional contribution margins from scheduled interventions. These interventions included valve procedures, ablation, revascularization and cardiac rhythm management $[25,37,38]$. In sum, the calculation can be expressed as HospContM $=$ HospDiagR - Cdiag * HospHFIncidence, where HospContM refers to hospital contribution margin, Hos$\mathrm{pDiagR}$ is diagnostic procedure reimbursement, Cdiag is cost of diagnostic test, and HospHFIncidence is the annual number of patients with HF treated within a hypothetical U.S. hospital system. Additional downstream effects of early HF diagnosis were also included in the hospital model, and can be summarized in two expressions: (1) HFhospC = ERHosp - PlannedHosp * (HospR HospC + NonR30dayHosp); and (2) Planned Interventions Revenue $=$ HospHFIncidence * Diagnostic Accuracy * Planned Procedure Rate. In these expressions, HFhospC refers to HF hospitalization cost, ERHosp is emergency hospitalization, PlannedHosp is planned hospitalization, HospR is hospital reimbursement, HospC is hospitalization cost and NonR30dayHosp is non-reimbursable 30-day hospitalization cost.

Procedural costs were equivalent to average payer reimbursement rates, which we conservatively estimated to be equivalent to U.S. Medicare rates. Table 3 provides further detail on clinical and cost input data for the hospital model. 
Table 3 Hospital model input data

\begin{tabular}{|c|c|c|c|}
\hline Parameter & $\mathrm{ECHO}$ & Fast-SENC Rest & Source \\
\hline Prevalence of Asymptomatic Heart Failure & $20 \%$ & $20 \%$ & Assumption \\
\hline Patients in High Risk Population to be Imaged & 3000 & 3000 & KOL interviews \\
\hline Stage B & $4.27 \%$ & $3.11 \%$ & {$[42]$} \\
\hline Stage C & $4.55 \%$ & $3.32 \%$ & {$[42]$} \\
\hline Stage $\mathrm{C}_{+}$ & $4.50 \%$ & $3.28 \%$ & Assumption \\
\hline $\begin{array}{l}\text { CMS and private payer ( } 90 \% \text { to } 10 \% \text { ratio) reimbursement } \\
\& \text { cost for HF hospitalization }\end{array}$ & $\$ 14,631$ & $\$ 14,631$ & {$[46]$} \\
\hline HF hospitalization cost from NIS 2015 sample & $\$ 7463$ & $\$ 7463$ & {$[47]$} \\
\hline 30 day HF rehospitalization & $26.90 \%$ & $26.90 \%$ & {$[43]$} \\
\hline HF hospitalization & $56.00 \%$ & N/A & {$[17]$} \\
\hline Imaging Per Year of Identified Asymptomatic HF Patients & 2 & 2 & Assumption \\
\hline ICD & $48 \%$ & $48 \%$ & {$[26]$} \\
\hline CRT & $20 \%$ & $20 \%$ & {$[26]$} \\
\hline Valve procedures & $4.5 \%$ & $4.5 \%$ & {$[37]$} \\
\hline Ablation procedures from tachycardia-induced cardiomyopathies & $0.79 \%$ & $0.79 \%$ & {$[48]$} \\
\hline Revascularizations for infarcted patients (previous MI) & $4.7 \%$ & $4.7 \%$ & {$[38]$} \\
\hline \multicolumn{4}{|l|}{ Total cost of ownership: } \\
\hline Acquisition cost (one time) & N/A & $\$ 40,000$ & MSI \\
\hline Maintenance cost (annual) & & $\$ 10,000$ & MSI \\
\hline Assumed medical equipment lifetime (years) & 10 & 10 & Assumption \\
\hline Cost per Test & N/A & $\$ 4.67$ & Calculation \\
\hline \multicolumn{4}{|l|}{ Imaging Modality Parameters: } \\
\hline Time Occupying Machine (min) & 30 & 12 & Hospital procedural guidelines \\
\hline Imaging Facility Cost Per Hour (Including Overhead, Wages, Bills, etc.) & $\$ 200$ & $\$ 320$ & Market research outcomes \\
\hline Test Inventory (Per Test) & $\$ 0$ & $\$ 150$ & MSI \\
\hline Cost of Contrast & $\$ 34$ & $\$ 0$ & CMS Fee Schedule 2017 \\
\hline Physician Reading Fee & $\$ 50$ & $\$ 0$ & Assumption \\
\hline \multicolumn{4}{|l|}{ Reimbursement Parameters: } \\
\hline Test Reimbursement & $\$ 231$ & $\$ 324$ & 2017 Medicare fee schedule \\
\hline Contrast Reimbursement & $\$ 34$ & $\$ 0$ & CMS 2017 \\
\hline
\end{tabular}

Note: $M S I=$ Myocardial Solutions Inc

\section{Results}

\section{Payer model}

The results of the payer model are shown in Table 4. Discounted total life-years and QALYs in the Fast-SENC cohort were 3.05 years, or 1.96 QALYs. In the ECHO arm, the total life-years were 1.71, 0.88 QALYs. Over their respective lifetimes, patients in the Fast-SENC cohort accumulated $\$ 24,647$ (discounted at $3 \%$ per year) in healthcare costs compared to substantially higher costs in the EHCO group $(\$ 39,097)$. Thus, the Fast-SENC cohort had lower costs and higher QALYs when compared to the ECHO cohort, and consequently Fast-SENC generated the largest cost savings difference in the first year, costing $72 \%$ less than the ECHO arm. This difference narrowed over the years, as the Fast-SENC arm accrued additional costs
Table 4 Payer Model Results

\begin{tabular}{lllll}
\hline Cumulative per Person & Fast-SENC & ECHO & Difference & $\begin{array}{l}\text { Percent } \\
\text { Difference }\end{array}$ \\
\hline 1-Year Horizon & $\$ 878$ & $\$ 3172$ & $(\$ 2294)$ & $-72 \%$ \\
3-year horizon & $\$ 15,031$ & $\$ 33,230$ & $(\$ 18,199)$ & $-55 \%$ \\
5-year horizon & $\$ 19,319$ & $\$ 36,526$ & $(\$ 17,208)$ & $-47 \%$ \\
10-year horizon & $\$ 19,154$ & $\$ 38,708$ & $(\$ 19,554)$ & $-51 \%$ \\
Lifetime & $\$ 24,647$ & $\$ 39,097$ & $(\$ 14,450)$ & $-37 \%$ \\
Life Years & 3.1 & 1.7 & 1.3 & $78 \%$ \\
QALY & 2.0 & 0.9 & 1.1 & $124 \%$ \\
Cost per QALY & $\$ 12,551$ & $\$ 44,623$ & $(\$ 32,071)$ & $-72 \%$ \\
Cost per LY & $\$ 8078$ & $\$ 22,845$ & $(\$ 14,767)$ & $-65 \%$ \\
ICER (SENC vs ECHO) & & Savings & $(\$ 13,288)$ & per QALY \\
\hline
\end{tabular}


resulting from a lower mortality rate, yielding a lifetime savings of 37\% for using Fast-SENC instead of ECHO. Fast-SENC's cost effectiveness is dominant to EHCO at all time horizons.

\section{Hospital model}

The results of the hospital model are presented in Table 5. The total cost per HF patient visit is $\$ 184$ for ECHO and \$209 for Fast-SENC, which results in hospital contribution margins of $\$ 81$ and $\$ 115$, respectively. Based on an assumption of 4200 procedures annually utilizing Fast-SENC, a hospital could achieve $\$ 481,600$ in total contribution margins. The Fast-SENC approach generates $\$ 238,600$ more in contribution margins when compared to ECHO annual utilization. In addition, Fast-SENC would allow clinicians to plan interventional procedures in advance instead of receiving HF patients in the emergency room. Fast-SENC is likely a more efficient use of resources for hospitals given its shorter exam time compared to ECHO, which allows for a significantly higher hourly profitability contribution. Fast-SENC generated a contribution margin of $\$ 573$ per hour of testing, while ECHO generated only $\$ 162$. As a result of its ability to identify early heart failure patients, Fast-SENC increases the number of scans the hospital will execute in the study patient population, as patients diagnosed with heart failure will require additional Fast-SENC tests to monitor and manage their condition.

\section{Sensitivity analysis}

We performed sensitivity analysis on key input parameters affecting the cost-effectiveness (payer model) of both patient cohorts. These parameters include, for example, Fast-SENC HF diagnostic accuracy, diagnosis of HF post HF hospitalization event for both patient cohorts, reduction in preventable hospitalization in the Fast-SENC group, Fast-SENC test reimbursement and the rate of HF diagnosis post HF hospitalization in the Fast-SENC group. One-way sensitivity analysis resulted in the tornado diagram shown in Fig. 2. Sensitivity analysis ranges were based on the parameter ranges reported in the literature search $[16,17,22,39]$. The results of univariate sensitivity analysis showed that the Fast-SENC strategy remained

Table 5 Hospital Model Results

\begin{tabular}{llll}
\hline Summary Metrics & ECHO & Fast-SENC & Margin gain \\
\hline $\begin{array}{l}\text { Hospital contribution margin } \\
\text { per HF imaging }\end{array}$ & $\$ 81$ & $\$ 115$ & $\$ 34$ \\
$\begin{array}{l}\text { Hospital HF test contribution } \\
\text { margin per hour }\end{array}$ & $\$ 162$ & $\$ 573$ & $\$ 411$ \\
$\begin{array}{l}\text { Annual hospital contribution } \\
\text { margin per HF imaging test }\end{array}$ & $\$ 24,300$ & $\$ 481,600$ & $\$ 238,600$ \\
\hline
\end{tabular}

robust for all parameters and did not surpass the threshold of $\$ 50,000$ per QALY.

\section{Discussion}

In this study, we performed a cost-effectiveness analysis to compare the currently available echocardiography strategy for assessing HF and a newly developed Fast-SENC CMR diagnostic approach. From a payer perspective, we found that Fast-SENC was associated with higher quality-adjusted life years $(1.96 \mathrm{v} .88)$ and, over a lifetime, patients in the Fast-SENC cohort accumulated $\$ 24,647$ per patient in expected healthcare costs compared to the higher costs in the ECHO group (\$39,097 per patient). In the first year, Fast-SENC, compared to $E C H O$, reduced costs by $72 \%$, although the cost-savings difference narrowed to $32 \%$ for the lifetime horizon. The driving factor of this apparent decrease in cost savings benefit is the extended lifetime and lower mortality of Fast-SENC arm patients leading to additional costs for care. By increasing the longevity and survival of patients, Fast-SENC's ability to identify heart failure before patients become symptomatic creates a significant opportunity to monitor and manage patients that otherwise would be identified by a cardiac event. By extrapolating these results to the entire US health system, we estimate the savings potential of Fast-SENC to be over $\$ 7$ billion annually increasing over time with $\$ 19$ billion in savings annually by 2030 . Actual savings potential could be higher when adjusted for private payer savings.

From the hospital perspective, a hospital can (on average, based on 4200 procedures annually utilizing Fast-SENC) generate $\$ 481,600$ in contribution margin, which is $\$ 238,600$ more compared to ECHO. The contribution derives from a combination of a higher profitability per test for Fast-SENC and an increased test volume for monitoring and managing heart failure patients identified by Fast-SENC but missed by ECHO. Fast-SENC's greater speed and accuracy is believed to impact hospitals and health plans by reducing the frequency of unnecessary procedures and identifying more patients who can benefit the most from more intensive treatment. Fast-SENC's ability to detect myocardial dysfunction in early heart failure patients can have other beneficial effects for hospitals, including increased ICU bed capacity through reduced HF hospitalizations, decreased 30-day HF patient re-hospitalizations, and better managed HF patients in need of more advanced interventions.

There are a few limitations in our study. The first limitation is the complexity of accurately depicting outcomes and resource use by disease state in simulation models. The second limitation pertains to input data, which were in majority retrieved from prospective 


\section{Incremental cost-effectiveness per quality-adjusted life year}

HF diagnosis post HF hosp Fast-SENC (3.11\%-50\%)

Fast-SENC reimbursement $(\$ 224-\$ 700)$

Reduction in preventable hosp for Fast-SENC (3\%-40\%)

HF diagnosis post HF hosp ECHO $(5 \%-56 \%)$

Fast-SENC diagnostic efficacy (50\%-100\%)

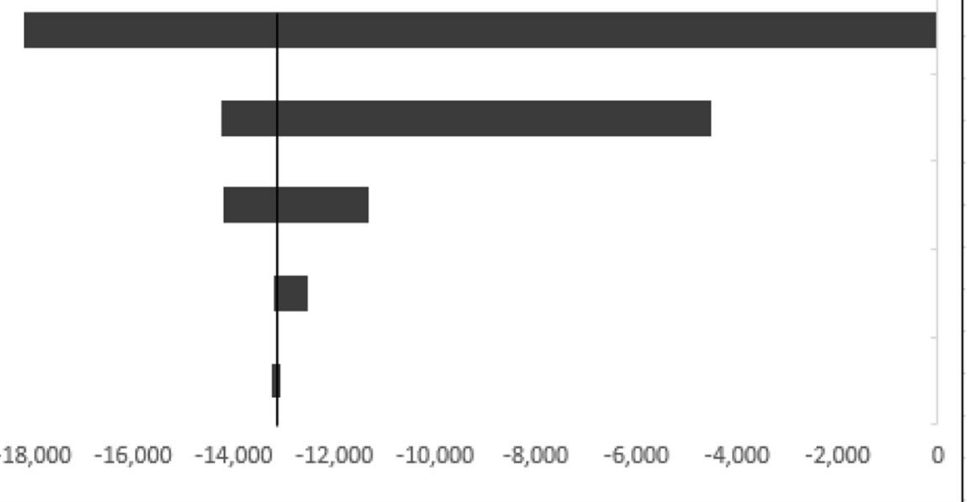

Fig. 2 Tornado diagram presenting results of univariate sensitivity analysis. The horizontal axis shows various incremental cost-effectiveness per quality-adjusted life year. At a threshold of $\$ 50,000$ per QALY, Fast-SENC strategy remains robust for all parameters

studies with inherent uncertainties. However, our sensitivity analysis attempted to identify influential factors and thus describe the scenarios in which our results would be valid. A third limitation is the assumption that disease transition probabilities for the Fast-SENC cohort are reduced because of earlier HF diagnosis and earlier treatment. Finally, based on the Michalsen et al. study that found that $54.2 \%$ of all hospitalizations are preventable with better patient management and lifestyle, we assume that Fast-SENCguided diagnosis could capture half of these hospitalizations (27.1\%) through improvement in HF detection and management, though it is possible that this assumption is imprecise. Finally, the assumption that the protective effects of earlier treatment are evident in later disease stages could also be considered a model limitation, as there is little literature based on longer-term longitudinal studies to directly support this assumption. Hence, we based this assumption on communications with clinical experts, who advised that the diagnostic effect could reasonably be tied to the treatment effect. The results are likely to be to some degree sensitive to this assumption.

Further clinical studies are needed to confirm our model assumptions. However, diagnosing HF earlier in the disease progression and treating patients accordingly is a well-studied area with a number of highquality published studies that conclude increased patient benefits and reduced mortality, similarly to our study. Despite these limitations, this study suggests that diagnostic tests enabling an early discovery of HF with appropriate treatments can be cost-effective if conditions that influence the economic impact of such tests are well evaluated.

\section{Conclusions}

Fast-SENC is associated with higher quality-adjusted life years and lower accumulated expected healthcare costs than echocardiogram patients. Fast-SENC also shows a significant short-term and lifetime cost-savings difference and a higher hospital contribution margin when compared to echocardiography. These results suggest that early discovery of heart failure with methods like Fast-SENC can be cost-effective when followed by the appropriate treatment.

\section{Additional file}

Additional file 1: Transition Matrix Calculations. (DOCX $15 \mathrm{~kb}$ )

\section{Abbreviations}

CMR: cardiac-tagged magnetic resonance imaging; ECHO: echocardiography; EF: ejection fraction; HF: heart failure; ISPOR: International Society for Pharmacoeconomics and Outcomes Research; LV: left ventricular; NICE: U.K. National Institute for Clinical Excellence; NYHA: New York Heart Association; QALY: quality adjusted life-years

\section{Acknowledgements}

N/A

\section{Funding}

The research was funded by a non-restricted grant from Myocardial Solutions, Inc., to Avalon Health Economics.

\section{Availability of data and materials}

All data are derived from published sources, with some data points for Fast SENC provided by Myocardial Solutions, Inc. All model data are retained by Avalon Health Economics. Avalon Health Economics is available for questions regarding the data, and all data used in the model are reported in the text and tables.

\section{Authors' contributions}

IS conducted data analysis and economic modelling; JS and IS shared responsibilities for writing. Both authors read and approved the final manuscript. 


\section{Authors' information}

N/A

\section{Competing interests}

The authors of this manuscript are full-time salaried employees of Avalon Health Economics and received no compensation specific to the production of this manuscript. The research was funded by a non-restricted grant from Myocardial Solutions, Inc., to Avalon Health Economics. The authors declare that they have no competing interests.

\section{Publisher's Note}

Springer Nature remains neutral with regard to jurisdictional claims in published maps and institutional affiliations.

\section{Received: 28 June 2018 Accepted: 1 April 2019}

Published online: 23 May 2019

\section{References}

1. Liu L, Eisen HJ. Epidemiology of heart failure and scope of the problem. Cardiol Clin. 2014;32(1):1-8 vii.

2. Zannad F, Agrinier N, Alla F. Heart failure burden and therapy. Europace : European pacing, arrhythmias, and cardiac electrophysiology : journal of the working groups on cardiac pacing, arrhythmias, and cardiac cellular electrophysiology of the European society of. Cardiology. 2009;11(Suppl 5):v1-9.

3. Weintraub WS, Cole J, Tooley JF. Cost and cost-effectiveness studies in heart failure research. Am Heart J. 2002;143(4):565-76.

4. Ambrosy AP, Fonarow GC, Butler J, Chioncel O, Greene SJ, Vaduganathan M, et al. The global health and economic burden of hospitalizations for heart failure: lessons learned from hospitalized heart failure registries. J Am Coll Cardiol. 2014;63(12):1123-33.

5. Guha K, McDonagh T. Heart failure epidemiology: European perspective. Curr Cardiol Rev. 2013;9(2):123-7.

6. Hodges P. Heart failure: epidemiologic update. Critical care nursing quarterly. 2009;32(1):24-32.

7. Vigen R, Maddox TM, Allen LA. Aging of the United States population: impact on heart failure. Curr Heart Fail Rep. 2012;9(4):369-74.

8. Cowie MR, Anker SD, Cleland JGF, Felker GM, Filippatos G, Jaarsma T, et al. Improving care for patients with acute heart failure: before, during and after hospitalization. ESC Heart Fail. 2014;1(2):110-45.

9. Liao L, Allen LA, Whellan DJ. Economic burden of heart failure in the elderly. PharmacoEconomics. 2008;26(6):447-62.

10. Bui AL, Horwich TB, Fonarow GC. Epidemiology and risk profile of heart failure. Nat Rev Cardiol. 2011;8(1):30-41.

11. Hollingworth W, Biswas M, Maishman RL, Dayer MJ, McDonagh T, Purdy S, et al. The healthcare costs of heart failure during the last five years of life: a retrospective cohort study. Int J Cardiol. 2016:224:132-8.

12. van Riet EES, Hoes AW, Wagenaar KP, Limburg A, Landman MAJ, Rutten FH. Epidemiology of heart failure: the prevalence of heart failure and ventricular dysfunction in older adults over time. A systematic review. Eur J Heart Fail. 2016;18(3):242-52.

13. Collins SP, Pang PS, Fonarow GC, Yancy CW, Bonow RO, Gheorghiade M. Is hospital admission for heart failure really necessary? the role of the emergency department and observation unit in preventing hospitalization and rehospitalization J Am Coll Cardiol. 2013;61(2):121-6.

14. Driscoll A, Meagher S, Kennedy R, Hay M, Banerji J, Campbell D, et al. What is the impact of systems of care for heart failure on patients diagnosed with heart failure: a systematic review. BMC Cardiovasc Disord. 2016;16(1):195.

15. Ziaeian B, Fonarow GC. The prevention of hospital readmissions in heart failure. Prog Cardiovasc Dis. 2016;58(4):379-85.

16. Michalsen A, Konig G, Thimme W. Preventable causative factors leading to hospital admission with decompensated heart failure. Heart. 1998 80(5):437-41.

17. Dunlay SM, Shah ND, Shi Q, Morlan B, VanHouten H, Long KH, et al. Lifetime costs of medical care after heart failure diagnosis. Circulation Cardiovascular quality and outcomes. 2011;4(1):68-75.

18. Choi EY, Rosen BD, Fernandes VR, Yan RT, Yoneyama K, Donekal S, et al. Prognostic value of myocardial circumferential strain for incident heart failure and cardiovascular events in asymptomatic individuals: the multiethnic study of atherosclerosis. Eur Heart J. 2013;34(30):2354-61.
19. Azaraksh A, Ivanov G, Bulanova N, Stazhadze L, Nikolaeva M, Vostrikov V CONTEMPORARY APPROACH TO DIAGNOSIS OF SUBCLINICAL HEART FAILURE. Georgian Med News. 2017;264:66-72.

20. Ciampi Q, Villari B. Role of echocardiography in diagnosis and risk stratification in heart failure with left ventricular systolic dysfunction. Cardiovasc Ultrasound. 2007:5(1):34.

21. Schuster A, Hor KN, Kowallick JT, Beerbaum P, Kutty S. Cardiovascular magnetic resonance myocardial feature tracking: concepts and clinical applications. Circulation Cardiovascular imaging. 2016;9(4):e004077.

22. Dunlay SM, Redfield MM, Weston SA, Therneau TM, Long KH, Shah ND, et al. Hospitalizations after heart failure diagnosis: a community perspective. Am Coll Cardiol. 2009;54(18):1695-702.

23. Zettler PJ, Fuse Brown EC. The challenge of paying for cost-effective cures. Am J Manag Care. 2017;23(1):62-4.

24. Cutler D, Ciarametaro M, Long G, Kirson N, Dubois R. Insurance switching and mismatch between the costs and benefits of new technologies. Am J Manag Care. 2017;23(12):750-7.

25. Kalogeropoulos AP, Georgiopoulou W, deFilippi CR, Gottdiener JS, Butler J. Echocardiography, natriuretic peptides, and risk for incident heart failure in older adults. the Cardiovascular Health Study JACC Cardiovascular imaging. 2012;5(2):131-40.

26. Kalogeropoulos AP, Samman-Tahhan A, Hedley JS, McCue AA, Bjork JB, Markham DW, et al. Progression to stage D heart failure among outpatients with stage $C$ heart failure and reduced ejection fraction. JACC Heart failure. 2017:5(7):528-37.

27. Ademi Z, Pasupathi K, Krum H, Liew D. Cost effectiveness of eplerenone in patients with chronic heart failure. American journal of cardiovascular drugs: drugs, devices, and other interventions. 2014;14(3):209-16.

28. Cao Q, Buskens E, Feenstra T, Jaarsma T, Hillege H, Postmus D. Continuoustime semi-Markov models in health economic decision making: an illustrative example in heart failure disease management. Medical decision making : an international journal of the Society for Medical Decision Making. 2016;36(1):59-71.

29. Clarke A, Pulikottil-Jacob R, Connock M, Suri G, Kandala NB, Maheswaran H, et al. Cost-effectiveness of left ventricular assist devices (LVADs) for patients with advanced heart failure: analysis of the British NHS bridge to transplant (BTT) program. Int J Cardiol. 2014;171(3):338-45.

30. Griffiths A, Paracha N, Davies A, Branscombe N, Cowie MR, Sculpher M. The cost effectiveness of ivabradine in the treatment of chronic heart failure from the U. K National Health Service perspective Heart (British Cardiac Society). 2014;100(13):1031-6.

31. Sandhu AT, Ollendorf DA, Chapman RH, Pearson SD, Heidenreich PA. Costeffectiveness of Sacubitril-valsartan in patients with heart failure with reduced ejection fraction. Ann Intern Med. 2016;165(10):681-9.

32. Sandhu AT, Goldhaber-Fiebert JD, Owens DK, Turakhia MP, Kaiser DW, Heidenreich PA. Cost-effectiveness of implantable pulmonary artery pressure monitoring in chronic heart failure. JACC Heart failure. 2016;4(5):368-75.

33. Woo CY, Strandberg EJ, Schmiegelow MD, Pitt AL, Hlatky MA, Owens DK, et al. Cost-effectiveness of adding cardiac resynchronization therapy to an implantable cardioverter-defibrillator among patients with mild heart failure. Ann Intern Med. 2015;163(6):417-26.

34. Yancy CW, Jessup M, Bozkurt B, Butler J, Casey DE, Drazner MH, et al. 2013 ACCF/AHA guideline for the Management of Heart Failure. J Am Coll Cardiol. 2013;62(16):e147-239.

35. Banz K. Cardiac resynchronization therapy (CRT) in heart failure--a model to assess the economic value of this new medical technology. Value in health : the journal of the International Society for Pharmacoeconomics and Outcomes Research. 2005:8(2):128-39.

36. Yancy CW, Jessup M, Bozkurt B, Butler J, Casey DE Jr, Colvin MM, et al. 2017 ACC/AHA/HFSA focused update of the 2013 ACCF/AHA guideline for the Management of Heart Failure: a report of the American College of Cardiology/ American Heart Association task force on clinical practice guidelines and the Heart Failure Society of America. Circulation. 2017;136(6):e137-e61.

37. De Sciscio P, Brubert J, De Sciscio M, Serrani M, Stasiak J, Moggridge GD. Quantifying the shift toward Transcatheter aortic valve replacement in lowrisk patients: a meta-analysis. Circulation Cardiovascular quality and outcomes. 2017;10(6).

38. Simoons ML, Windecker S. Controversies in cardiovascular medicine: chronic stable coronary artery disease: drugs vs. revascularization. Eur Heart J. 2010; 31(5):530-41.

39. services CMM. Medicare Fee Schedule Federal Register. 2017;2016. 
40. Siegel JED, The members of the panel on cost effectiveness in $\mathrm{H}$, medicine, Torrance GW, Russell LB, Luce BR, et al. Guidelines for Pharmacoeconomic studies : recommendations from the panel on cost effectiveness in health and medicine. PharmacoEconomics. 1997:11(2):159-68.

41. Kane GC, Karon BL, Mahoney DW, Redfield MM, Roger VL, Burnett JC Jr, et al. Progression of left ventricular diastolic dysfunction and risk of heart failure. Jama. 2011;306(8):856-63.

42. Pfeffer MA, Braunwald E, Moye LA, Basta L, Brown EJ Jr, Cuddy TE, et al. Effect of captopril on mortality and morbidity in patients with left ventricular dysfunction after myocardial infarction. Results of the survival and ventricular enlargement trial. The SAVE investigators. N Engl J Med. 1992;327(10):669-77.

43. Jencks SF, Williams MV, Coleman EA. Rehospitalizations among patients in the Medicare fee-for-service program. N Engl J Med. 2009;360(14):1418-28.

44. Obi EN, Swindle JP, Turner SJ, Russo PA, Altan A. Health care costs for patients with heart failure escalate nearly 3 -fold in final months of life. Journal of managed care \& specialty pharmacy. 2016;22(12):1446-56.

45. Wang G, Zhang Z, Ayala C, Wall HK, Fang J. Costs of heart failure-related hospitalizations in patients aged 18 to 64 years. Am J Manag Care. 2010; 16(10):769-76

46. Kilgore M, Patel HK, Kielhorn A, Maya JF, Sharma P. Economic burden of hospitalizations of Medicare beneficiaries with heart failure. Risk management and healthcare policy. 2017;10:63-70.

47. Akintoye E, Briasoulis A, Egbe A, Orhurhu V, Ibrahim W, Kumar K, et al. Effect of hospital ownership on outcomes of heart failure hospitalization. Am J Cardiol. 2017:120(5):831-7.

48. Kneeland PP, Fang MC. Trends in catheter ablation for atrial fibrillation in the United States. J Hosp Med. 2009:4(7):E1-5.

Ready to submit your research? Choose BMC and benefit from:

- fast, convenient online submission

- thorough peer review by experienced researchers in your field

- rapid publication on acceptance

- support for research data, including large and complex data types

- gold Open Access which fosters wider collaboration and increased citations

- maximum visibility for your research: over $100 \mathrm{M}$ website views per year

At $\mathrm{BMC}$, research is always in progress.

Learn more biomedcentral.com/submissions 